\title{
Association Between IL6R Polymorphisms and Cachexia Phenotype in Patients with Pancreatic Ductal Adenocarcinoma
}

\author{
Nicholas J. Polster, Joseph E. Rupert, Andrew R. Young, Teresa A. Zimmers \\ Indiana University School of Medicine Department of Surgery
}

Background: Cachexia, manifested as progressive adipose and muscle wasting, affects up to $80 \%$ of patients with pancreatic ductal adenocarcinoma (PDAC) and significantly increases morbidity and mortality. Increased inflammation is an underlying mechanism in almost all cases of cachexia. Trans-signaling of Interleukin-6 (IL-6) via the soluble form of its receptor (sIL6R) has been shown to promote inflammation. Certain polymorphisms of the IL6R gene such as rs2228145 (Asp358Ala substitution) are associated with increased levels of sIL6R. We hypothesize that patients with PDAC possessing IL6R alleles correlated with higher levels of circulating sIL6R will have increased systematic inflammation manifested as increased cachexia prevalence or severity.

Methods: DNA was extracted from prospectively collected blood samples acquired from patients with PDAC and from non-cancer controls. Genotype at the rs2228145 polymorphism was determined by TaqMan qPCR genotyping. The resulting genotypes $(A / A, A / C, C / C)$ were compared against cachexia-related metrics, including presence of cachexia ( $>5 \%$ body weight loss in the prior 6 months), body mass index (BMI), BMI-adjusted weight loss grade (BMI-WLG), and muscle and adipose volumes calculated from height-adjusted surface areas obtained from CT scans at the level of the third lumbar vertebra.

Results: $83.3 \%$ of patients with PDAC heterozygous (A/C) and $84.6 \%$ of the patients homozygous for the rs2228145 polymorphism (C/C) exhibited cachexia, versus $57.9 \%$ of patients homozygous for the reference allele $(A / A),(P=0.0364$, Chi-square test). No significant difference was found among genotypes for BMI, 6-month weight loss, BMI-WL grade, or muscle and adipose tissue indices.

Conclusion: Patients with PDAC who are possess at least one copy of the rs2228145 polymorphism have a higher incidence of cachexia than those who are homozygous for the reference allele. This association suggests a causal role for slL6R in cancer cachexia. 